\title{
ON THE CLOSURE-PRESERVING SUM THEOREM
}

\author{
M. K. SINGAL AND SHASHI PRABHA ARYA
}

ABSTRACT. The closure-preserving sum theorem holds for a property $\mathcal{P}$ if the following is satisfied: "If $\left\{F_{a}: a \in \Omega\right\}$ is a hereditarily closurepreserving closed covering of $X$ such that each $F_{\alpha}$ possesses the property $\mathcal{P}$, then $X$ possesses $\mathcal{P}$ ". A general technique for proving this theorem is developed. The theorem is shown to hold for a large number of topological properties. As an application, three general sum theorems have also been obtained.

1. Introduction. A family $\mathfrak{Q}=\left\{A_{\alpha}: \alpha \in \Omega\right\}$ of subsets of a space $X$ is said to be hereditarily closure-preserving (to be abbreviated as H-closurepreserving if every family $B=\left\{B_{\alpha}: \alpha \in \Omega\right\}$ such that $B_{\alpha} \subset A_{\alpha}$ for each $a \epsilon$ $\Omega$ is closure-preserving. Let $\mathcal{P}$ be any topological property. We say that the closure-preserving sum theorem (to be abbreviated as CPST) holds for $\mathcal{P}$ if the following is satisfied:

"If $\left\{F_{\alpha}: \alpha \in \Omega\right\}$ is an H-closure-preserving closed covering of $X$ such that each $F_{a}$ possesses the property $\mathcal{P}$, then $X$ possesses $\mathcal{P}$ ".

The above theorem with "H-closure preserving" replaced by "locally finite" is known as the locally finite sum theorem. The locally finite sum theorem has been of interest to many people since it holds for a large number of interesting properties. Many interesting consequences of this theorem have been noted by the authors in a series of recent papers [11], [12], [13]. It is the purpose of the present note to show that the CPST holds for a large number of topological properties. A general technique for proving the CPST will be obtained. In particular, it will be shown that the CPST holds for all those properties which are preserved under disjoint sums and also under closed continuous maps. It will also be shown that if the CPST holds for a property $\mathcal{P}$, then it also holds for the property hereditarily $\mathcal{P}$. As an application of the CPST, three general sum theorems will be obtained. When applied to all those properties for which the CPST holds and which are closed hereditary, these theorems offer many new results and many results known already are improved.

Received by the editors June 6, 1974 .

AMS (MOS) subject classifications (1970). Primary 54D99; Secondary 54D15, $54 \mathrm{D} 20$.

Key words and phrases. Closure-preserving, hereditarily closure-preserving, strongly hereditarily closure-preserving, $\sigma$-locally finite, $\sigma$-hereditarily closure-preserving, $\sigma$-strongly hereditarily closure-preserving.

Copyright $@$ 1975. American Mathematical Society 
2. The closure-preserving sum theorem.

2.1 Theorem. Let $\mathcal{P}$ be a topological property such that:

(a) the disjoint sum of spaces possessing the property $\mathcal{P}$ possesses $\mathcal{P}$;

(b) $\mathcal{P}$ is preserved under closed continuous maps. Then the CPST holds for the property $\mathcal{P}$.

Proof. Let $\left\{F_{a}: \alpha \in \Omega\right\}$ be an H-closure-preserving closed covering of a space $X$ such that each $F_{a}$ possesses the property $\mathcal{P}$. For each $a \in \Omega$, let $K_{a}$ denote a copy of $F_{a}$ and let $f_{a}$ be this homeomorphism. Let $X^{*}$ denote the disjoint topological sum of $K_{a}^{\prime}$ s. Let $f: X^{*} \rightarrow X$ be the mapping defined as follows: for each $x \in X^{*}, f(x)=f_{\alpha}(x)$ if $x \in K_{a}$. It is easy to verify that $f$ is a closed continuous mapping. In view of hypothesis (a), $X^{*}$ possesses the property $\mathcal{P}$. Again, $f$ being closed and continuous, $X$ possesses the property $\mathcal{P}$ in view of hypothesis (b), hence, the result.

2.2. Remark. We mention below those properties which are preserved under disjoint sums and are also preserved under closed continuous maps (some of these are preserved even under quotients): Normality, perfect normality, collectionwise normality [2], complete normality, paracompactness + Hausdorff property, pointwise paracompactness, subparacompactness [5], msubparacompactness [14], stratifiability [3], [4], semistratifiability [6], the property of being a normal $T_{1} \sigma$-space [10], local connectedness, local pathwise connectedness, the properties of being a $P$-space (that is, every $G_{\delta}$ set is open), a chain net space [8], a $k$-space, a sequential space [7], a Fréchet space, a c-space [9].

In view of Theorem 2.1 above, the CPST follows for all properties of topological spaces mentioned above.

2.3 Theorem. If the CPST holds for a property $\mathcal{P}$, then it also holds for the property hereditarily $P$.

Proof. Let $\left\{F_{a}: a \in \Omega\right\}$ be an H-closure-preserving closed covering of a space $X$ such that each $F_{a}$ possesses the property $\mathcal{P}$ hereditarily. Let $A$ be any subset of $X$. We shall prove that $\left\{A \cap F_{a}: a \in \Omega\right\}$ is H-closurepreserving relative to $A$. Let $\left\{U_{\alpha}: \alpha \in \Omega\right\}$ be any family of subsets of $A$ such that $U_{a} \subset A \cap F_{a}$ for each $a \in \Omega$.

Let $\Omega^{\circ}$ be any subset of $Q$. Then

$$
\begin{aligned}
\bigcup\left\{\mathrm{cl}_{A} U_{\alpha}: \alpha \in \Omega^{\prime}\right\} & =\bigcup\left\{A \cap \operatorname{cl} U_{\alpha}: \alpha \in \Omega^{\prime}\right\}=A \cap\left(\bigcup\left\{\mathrm{cl} U_{a}: \alpha \in \Omega^{\prime}\right\}\right) \\
& =A \cap \operatorname{cl}\left[\bigcup\left\{U_{\alpha}: \alpha \in \Omega^{\prime}\right\}\right] \\
& \left(\left\{U_{\alpha}: \alpha \in \Omega\right\}\right. \text { being closure-preserving) } \\
& =\operatorname{cl}_{A}\left[\bigcup\left\{U_{\alpha}: \alpha \in \Omega^{\prime}\right\}\right] .
\end{aligned}
$$


Hence $\left\{A \cap F_{\alpha}: \alpha \in \Omega\right\}$ is an H-closure-preserving closed (relative to $A$ ) covering of $A$. Since each $F_{a}$ possesses the property $\mathcal{P}$ hereditarily, therefore each $A \cap F_{\text {a }}$ possesses $\mathcal{P}$. Since CPST holds for $\mathcal{P}$, therefore $A$ possesses $\mathcal{P}$. Hence $X$ possesses the property $\mathcal{P}$ hereditarily.

2.4. Remark. In view of Theorem 2.3 the CPST also holds for the following properties of topological spaces: Complete collectionwise normality, hereditarily paracompactness + the Hausdorff property, hereditarily pointwise paracompactness, hereditarily subparacompactness, hereditarily thsubparacompactness, hereditarily local connectedness, hereditarily pathwise connectedness, the property of being hereditarily a $k$-space, or hereditarily a Frechét space, or hereditarily a sequential space, or hereditarily a cospace, or hereditarily a chain net space.

3. Applications. Let $\mathbb{A}=\left\{A_{\alpha}: \alpha \in \Omega\right\}$ be any family of subsets of a space $X$. We say that $\mathscr{A}$ is strongly hereditarily closure-preserving (to be abbreviated as SH-closure-preserving) if the family $\left\{\operatorname{cl}_{a}: \alpha \in \Omega\right\}$ is $\mathrm{H}$ closure-preserving.

Every locally finite family is obviously SH-closure-preserving.

Let $\mathcal{P}$ denote any topological property for which the CPST holds and which is closed hereditary.

3.1 Theorem. Let $\mathcal{O}$ be a $\sigma$-SH-closure-preserving open covering of a space $X$ such that $\mathrm{cl} V$ possesses the property $\mathcal{P}$ for each $V \in \mathcal{O}^{\mathcal{O}}$. Then $X$ possesses $\mathcal{P}$.

Proof. Let $O=\bigcup_{i=1}^{\infty} \mho_{i}$ where each ()$_{i}$ is SH-closure-preserving. For each positive integer $i$, let $V_{i}=\bigcup\left\{V: V \in \mathcal{O}_{i}\right\}$. Then $\left\{\operatorname{cl} V: V \in \mathcal{O}_{i}\right\}$ is $\mathrm{H}$ closure-preserving. Thus $\left\{\mathrm{cl} V: V \in \mathcal{O}_{i}\right\}$ is an H-closure-preserving closed covering of $\mathrm{cl} V_{i}$, each element of which possesses the property $\mathcal{P}$. Then cl $V_{i}$ possesses $\mathcal{P}$, since CPST holds for $\mathcal{P}$. Now let $F_{1}=\operatorname{cl} V_{1}$ and, for $i \geq 2$, let $F_{i}=\operatorname{cl} V_{i} \sim \bigcup\left\{V_{j}: j<i\right\}$. If $x \in X$, there exists a positive integer $j$ such that $x \in V_{j}$. For each $i>j, V_{j} \cap F_{i}=\varnothing$. Since $V_{j}$ is open, it follows that $\left\{F_{i}: i=1,2, \ldots\right\}$ is locally finite and, hence, H-closure-preserving. Since $\mathcal{P}$ is closed hereditary, therefore each $F_{i}$ possesses the property $\mathcal{P}$. Thus $\left\{F_{i}: i=1,2, \ldots\right\}$ is an $\mathrm{H}$-closure-preserving closed covering of $X$, each element of which possesses the property $\mathcal{P}$. Since CPST holds for $\mathcal{P}$, therefore $X$ must possess $\mathcal{P}$.

3.2 Corollary. If 7 is a $\sigma$-locally finite open covering of a space $X$ such that $\mathrm{cl} B$ possesses the property $\mathcal{P}$ for each $V \in \mathcal{O}$, then $X$ possesses $\mathcal{P}$.

3.3 Theorem. Let $X$ be a regular space and let $(7)$ be a $\sigma$-SH-closurepreserving open covering of $X$, every element of which possesses $\mathcal{P}$. If frontier $V$ is a-paracompact [1] for each $V \in \mathcal{Y}$, then $X$ possesses $P$. 
Proof. Since frontier $V$ is $\alpha$-paracompact and $X$ is regular, there exists a locally finite open (in $X$ ) covering $\left\{U_{\alpha}: a \in \Omega\right\}$ of frontier $V$ such that each $\mathrm{cl} U_{a}$ is contained in some member of 0 . For each $\alpha \in \Omega$, let $W_{\alpha}=$ $\operatorname{cl} U_{a} \cap \operatorname{cl} V$ and $W_{0}=\operatorname{cl} V \sim \bigcup_{a \in \Omega} U_{a}$. It follows that $W_{0}$ and each $W_{a}$ possess property $\mathcal{P}$. Thus $\left\{W_{0}\right\} \cup\left\{W_{a}: \alpha \in \Omega\right\}$ is a locally finite and, hence, an $\mathrm{H}$-closure-preserving closed covering of $\mathrm{cl} V$, every element of which possesses the property $\mathcal{P}$. It follows that $\mathrm{cl} V$ possesses $\mathcal{P}$. Hence, $X$ possesses $\mathcal{P}$ in view of Theorem 3.1 .

3.4 Corollary. Let $X$ be a regular space and 0 a $\sigma$-SH-closure-preserving open covering of $X$ such that each $V \in \mathcal{O}$ possesses the property $\mathcal{P}$. Then $X$ possesses $\mathcal{P}$ if frontier $V$ is compact for each $V \in \mathcal{O}$.

3.5 Corollary. Let $X$ be a regular space and 0 a $\sigma$-locally finite open covering of $X$ such that each $V \in \mathcal{O}$ possesses the property $\mathcal{P}$. Then $X$ possesses $\mathcal{P}$ if frontier $V$ is compact for each $V \in \mathcal{O}$.

An open subset $V$ of a space $X$ is said to be an elementary set if $V=$ $\bigcup_{i=1}^{\infty} V_{i}$ where each $V_{i}$ is open and $\mathrm{cl} V_{i} \subset V$ for each $i$.

3.6 Theorem. If $\mathrm{U}$ is a $\sigma$-H-closure preserving elementary covering of $X$ such that each $U \in U$ possesses the property $\mathcal{P}$, then $X$ possesses $\mathcal{P}$.

Proof. Let $\mathbb{U}=\bigcup_{i=1}^{\infty} U_{i}$ where each $U_{i}=\bigcup\left\{U_{a}: a \in \Omega_{i}\right\}$ is H-closurepreserving. For each $\alpha \in \Omega_{i}, U_{a}=\bigcup_{j=1}^{\infty} U_{a_{j} j}$ where each $U_{a, j}$ is an open

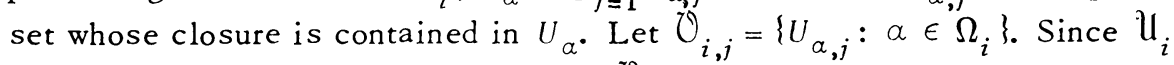
is $\mathrm{H}$-closure-preserving, it follows that $\mathcal{O}_{i, j}$ is SH-closure-preserving. If $\mathcal{O}=\bigcup_{i=1}^{\infty} \bigcup_{j=1}^{\infty} \bigcup_{i, j}$, then $\vartheta$ is a $\sigma$-SH-closure-preserving open covering of $X$ such that the closure of each member of $\mathcal{O}$ possesses the property $\mathcal{P}$. Hence $X$ possesses $\mathcal{P}$ in view of Theorem 3.1.

3.7 Corollary. If $\mathrm{U}$ be a $\sigma$-locally finite elementary covering of $X$ such that each $U \in \mathbb{U}$ possesses the property $\mathcal{P}$, then $X$ possesses $\mathcal{P}$.

3.8 Remark. It has been pointed out in Remarks 2.2 and 2.4 that the CPST holds for a large number of properties. We list below those properties out of these which are at least closed hereditary, so that Theorems 3.1, 3.3 and 3.6 and Corollary 3.4 are applicable to all these properties: Normality, perfect normality, collectionwise normality, complete normality, paracompactness + Hausdorff property, pointwise paracompactness, subparacompactness, m-subparacompactness, stratifiability, semistratifiability, the properties of being a normal $T_{1} \sigma$-space, a $\mathcal{P}$-space, a chain net space, a $c$-space, a $k$ space, a Fréchet space, and a sequential space.

In view of Theorem 2.3, we have the following corollaries to Theorems 3.1, 3.3 and 3.6 for any property $\mathcal{P}$ for which the CPST holds: 
3.9 Corollary. If 7 is a $\sigma$-SH-closure-preserving open covering of a space $X$ such that $\mathrm{cl} V$ possesses the property $\mathcal{P}$ hereditarily for each $V \in \mathcal{O}$, then $X$ possesses $\mathcal{P}$-hereditarily.

3.10 Corollary. If $X$ is regular and $\mathcal{O}$ is a $\sigma$-SH-closure-preserving open covering of $X$ such that each $V \in \mathcal{O}$ possesses the property $\mathcal{P}$ hereditarily and frontier $V$ is a-paracompact (or compact) for each $V \in \mathcal{O}$, then $X$ possesses the property $\mathcal{P}$ hereditarily.

3.11 Corollary. If 7 be a $\sigma-H$-closure-preserving elementary covering of $X$ such that each $V \in \mathcal{O}$ possesses the property $\mathcal{P}$ hereditarily, then $X$ possesses the property $\mathcal{P}$ bereditarily.

Corollaries 3.7, 3.10 and 3.11 are applicable to all properties $\mathcal{P}$ in Remark 2.2. The authors are grateful to the referee for his valuable suggestions.

\section{REFERENCES}

1. C. E. Aull, Paracompact subsets, General Topology and its Relations to Modern Analysis and Algebra, II (Proc. Second Prague Topological Sympos., 1966), Academia, Prague, 1967, pp. 45-51. MR 38 \#2737.

2. R. H. Bing, Metrization of topological spaces, Canad. J. Math. 3 (1951), 175186. MR 13, 264.

3. C. J. R. Borges, On stratifiable spaces, Pacific J. Math. 17 (1966), 1-16. MR $32 \# 6409$.

4. - Stratifiable spaces and continuous extensions, Topology Conf., Arizona State University, Tempe, Ariz., 1967, pp. 37-54.

5. D. K. Burke, On subparacompact spaces, Proc. Amer. Math. Soc. 23 (1969), 655-663. MR $40 \# 3508$.

6. G. D. Creede, Semi-stratifiable spaces, Topology Conf., Arizona State University, Tempe, Ariz., 1967, pp. 318-323.

7. S. P. Franklin, Spaces in which sequences suffice, Fund. Math. 57 (1965), 107-115. MR 31 \#5184.

8. H. Herrlich, Quotienten geordneter Räume and Folgenkonvergenz, Fund. Math. 61 (1967), 79-81. MR $36 \# 4528$.

9. R. C. Moore and S. G. Mrowka, Topologies determined by countable objects, Notices Amer. Math. Soc. 11 (1964), 554. Abstract \#614-88.

10. A. Okuyama, Some generalizations of metric spaces, their metrization theorems and product spaces, Sci. Rep. Tokyo Kyoiku Daigaku Sect. A 9 (1968), 236254. MR 37 \#5846.

11. M. K. Singal and Shashi Prabha Arya, Two sum theorems for topological spaces, Israel J. Math. 8 (1970), 155-158. MR 41 \#7612.

12. - - More sum theorems for topological spaces, Pacific J. Math. 59 (1975).

13. - A note on the locally finite sum theorem, Mathematika 19 (1972), 121-127. MR $47 \# 9511$.

14. M. K. Singal and Pushpa Jain, M-subparacompact spaces, Jñānabha 2 (1972), 143-158.

DEPARTMENT OF MATHEMATICS, MEERUT UNIVERSITY, MEERUT, INDIA 250001 (Current address of M. K. Singal)

MAITREYI COLLEGE, UNIVERSITY OF DELHI, DELHI, INDIA

Current address (S.P.Arya): Department of Mathematics, Somerville College, Oxford, OX2 6HD United Kingdom 